\title{
Universiteit
}

Leiden

The Netherlands

\section{Observation of Orbital Angular Momentum Sidebands due to optical reflection}

Löffler, W.; Aiello, A.; Woerdman, J.P.

\section{Citation}

Löffler, W., Aiello, A., \& Woerdman, J. P. (2012). Observation of Orbital Angular Momentum Sidebands due to optical reflection. Physical Review Letters, 109(11), 113602. doi:10.1103/PhysRevLett.109.113602

Version: $\quad$ Not Applicable (or Unknown)

License: $\quad$ Leiden University Non-exclusive license

Downloaded from: https://hdl.handle.net/1887/64255

Note: To cite this publication please use the final published version (if applicable). 


\title{
Observation of Orbital Angular Momentum Sidebands due to Optical Reflection
}

\author{
W. Löffler, ${ }^{1, *}$ Andrea Aiello, ${ }^{2,3}$ and J. P. Woerdman ${ }^{1}$ \\ ${ }^{1}$ Huygens Laboratory, Leiden University, P.O. Box 9504, 2300 RA Leiden, Netherlands \\ ${ }^{2}$ Max Planck Institute for the Science of Light, Günther-Scharowsky-Straße 1/Building 24, 91058 Erlangen, Germany \\ ${ }^{3}$ Institute for Optics, Information and Photonics, Universität Erlangen-Nürnberg, Staudtstraße 7/B2, 91058 Erlangen, Germany
}

(Received 13 April 2012; published 13 September 2012)

\begin{abstract}
We investigate how the orbital angular momentum of a paraxial light beam is affected upon reflection at a planar interface. Theoretically, the unavoidable angular spread of the beam leads to orbital angular momentum sidebands, which are found to be already significant for a modest beam spread (0.05). In analogy to the polarization Fresnel coefficients, we develop an analytical theory based upon spatial Fresnel coefficients; this allows a straightforward prediction of the strength of the sidebands. We confirm this by experiment and numerical simulation.
\end{abstract}

A light beam, either classical or quantum, possesses spatially transverse degrees of freedom. A very popular example is the orbital angular momentum (OAM) of light [1]; this has important applications in quantum communication [2]. The key advantage of OAM for quantum communication is its high dimensionality; this allows a single photon to carry much more $[3,4]$ than the single bit of information (qubit) enabled by the polarization degree of freedom. In this Letter, we investigate, theoretically and experimentally, the effect of optical reflection at a planar interface on the OAM state of a beam. This is a relevant issue, since reflection is the simplest possible optical operation, and is often unavoidable in experiments and applications. An analogous issue is well known for the case of light's polarization; this is generally strongly affected by Fresnel reflection (apart from special cases [5]). What happens in the OAM case? Everyday experience tells us that image distortion does not occur if we use a planar mirror; this suggests that the spatial state of the input beam (and thus also the OAM spectrum) should be well preserved upon reflection.

As we will see, the OAM state is indeed conserved if we use an ideal mirror, which we define as a (planar) mirror with infinite dielectric contrast. However, a practical mirror has a finite dielectric contrast; think for instance of a single dielectric interface, or a multilayer dielectric mirror, or a metal mirror. In this case, wave optics leads to diffractive corrections upon Snell's reflection law such as the Goos-Hänchen (GH) [6] and Imbert-Fedorov (IF) [7,8] shifts: the reflected beam is shifted relative to the geometrical optics reflected ray [9-13]. Consequently, the spatial state of light is modified by reflection; we describe this theoretically by spatial Fresnel coefficients that act upon the transverse modes of the incident light beam, analogous to the conventional polarization Fresnel coefficients that act upon the polarization modes. We find that the reflection-induced modification of the OAM state depends on the angular spread of the beam; this leads to OAM sidebands, even in the paraxial approximation. This is confirmed by our experimental results.

Our work connects with several papers that report on OAM spectral broadening due to (unintentional) experimental misalignment of the reference frame with respect to the OAM beam $[4,14,15]$. Such broadening can obviously be removed by proper readjustment of the setup to compensate the reference misalignment. However, in our case the misalignment is intrinsic to reflection (due to $\mathrm{GH}$ and IF shifts) and (as we will see) depends on the spatial OAM mode. Therefore, it cannot be cancelled simply by optical adjustment if we deal with a superposition of spatial modes as input states. We aim here to quantify the corresponding OAM spectral broadening. Our work also connects with that of Okuda and Sasada [16,17] who study giant (nonperturbative) deformation of an OAM mode due to total internal reflection for incidence very close to the critical angle. In such a singular regime, beam shifts (GH and IF), and thus the OAM mode spectrum, are ill-defined concepts [17]; we do not consider this singular total internal reflection case in the present Letter. Finally, there is also a connection with very recent work on the weak measurement aspects and topology of vortex singularities occurring in planar reflection $[18,19]$.

Theoretically, we describe the reflection process in terms of a scattering operator $\hat{S}=\sum_{\lambda} \hat{P}_{\lambda} \otimes \hat{M}_{\lambda}$, where $\hat{P}_{\lambda}$ acts on the polarization state $|\lambda\rangle[\lambda=1$ and $\lambda=2$ correspond to $p$ (in plane) and $s$ (out of plane) polarization, respectively] and $\hat{M}_{\lambda}$ on the spatial state $|\psi\rangle$. Here, we have adopted a quantum notation for the sake of clarity. We restrict ourselves to a paraxial light field; in this case, polarization and spatial degree of freedom factorize: $|i n\rangle=|\lambda\rangle|\psi\rangle$. Upon reflection, $\hat{S}$ mixes the polarization and spatial part; thus, after scattering, it is not possible to write the state as before in a product of polarization times spatial state $[12,20]$; this establishes a link between beam shifts and weak measurements $[10,19]$. 
In more detail, we discuss the incoming field $\mathbf{E}_{i}\left(x_{i}, y_{i}, z_{i}, t\right)=\operatorname{Re}\left[\mathbf{A}_{i}\left(x_{i}, y_{i}, z_{i}\right) \exp (-i \omega t)\right]$ in terms of its analytic signal [21-23] $\mathbf{A}_{i}\left(x_{i}, y_{i}, z_{i}\right)=$ $\sum_{\lambda} a_{\lambda} \hat{\mathbf{e}}_{\lambda}^{\prime} \psi\left(x_{i}, y_{i}, z_{i}\right)$, where $\psi$ describes the spatial shape of the beam (which we keep arbitrary at this point), $\hat{\mathbf{e}}_{1}^{\prime}=\hat{\mathbf{x}}_{i}$ and $\hat{\mathbf{e}}_{2}^{\prime}=\hat{\mathbf{y}}_{i}$ are the incoming-beam unit vectors, and $a_{1,2}$ are the polarization coefficients. All coordinates are expressed in units of $k$ and are consequently dimensionless. The reflected field can be written as

$$
\mathbf{A}(x, y, z)=\sum_{\lambda} a_{\lambda} r_{\lambda}(\theta) \psi\left(-x+X_{\lambda}, y-Y_{\lambda}, z\right) \hat{\mathbf{e}}_{\lambda},
$$

where $\hat{\mathbf{e}}_{1}=\hat{\mathbf{x}}_{r}$ and $\hat{\mathbf{e}}_{2}=\hat{\mathbf{y}}_{r}$ are the unit vectors in the reflected-beam coordinate system (Fig. 1). The reflected field $\mathbf{A}$ depends on the Fresnel reflection coefficients $r_{\lambda}$ and the four complex beam shifts $X_{\lambda}$ and $Y_{\lambda}$, whose real (imaginary) part corresponds to spatial (angular) longitudinal Goos-Hänchen [6] and transverse Imbert-Fedorov [8,9] shifts, respectively [22]: $X_{\lambda}=-i \partial_{\theta}\left[\ln r_{\lambda}(\theta)\right]$ and $Y_{1}=-i \frac{a_{2}}{a_{1}}\left(1+\frac{r_{2}}{r_{1}}\right) \cot \theta, Y_{2}=i \frac{a_{1}}{a_{2}}\left(1+\frac{r_{1}}{r_{2}}\right) \cot \theta$. By analyzing $X_{\lambda}$ and $Y_{\lambda}$, it can easily be seen that only an infinite refractive index contrast makes them disappear, so, only at reflection from such a perfect mirror, the reflected mode $\mathbf{A}(x, y, z)$ is not perturbed. Since the combined shift $\mathbf{R}_{\lambda}=$ $\left(X_{\lambda}, Y_{\lambda}\right)$ is supposedly small, we can Taylor-expand the shifted function $\psi$ to find deviations from geometrical optics reflection. We obtain for the spatial part with $\mathbf{R}=(x, y)$

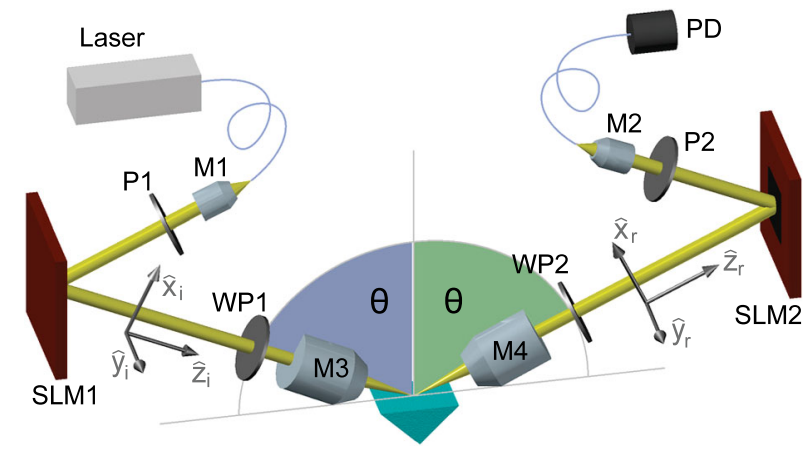

FIG. 1 (color online). Experimental setup: Light from a single mode fiber pigtailed laser is collimated $(M 1)$, polarized $(P 1)$, and then modulated using a phase-only spatial light modulator (SLM1) to prepare a certain mode. This light is then reflected at the hypotenuse of a glass prism, and the reflected light is analyzed using a combination of SLM2 and a single mode fiber connected to a photodiode (PD). Microscopy objectives $M 3$ and $M 4(10 \times$, numerical aperture 0.25$)$ can be introduced to change the beam focussing $\left(\theta_{0}\right)$, and $\lambda / 2$ wave plates WP1 and WP2 are used to modulate the polarization. The setup is symmetric relative to the prism, with distances $M 1-S L M 1=55 \mathrm{~cm}$ and SLM1-SLM2 $=148 \mathrm{~cm}$.

$$
\begin{aligned}
\left\langle x, y, z\left|\hat{M}_{\lambda}\right| \psi\right\rangle & =\psi\left(-x+X_{\lambda}, y-Y_{\lambda}, z\right) \\
& \simeq \psi(-x, y, z)+\mathbf{R}_{\lambda} \cdot \frac{\partial}{\partial \mathbf{R}} \psi(-x, y, z) .
\end{aligned}
$$

Now, we specialize to a specific mode basis and expand the spatial part of the input field in terms of the Laguerre-Gauss (LG) modes $\phi_{\ell}^{p}$ as $|\psi\rangle=\sum_{\ell, p}\left|\phi_{\ell}^{p}\right\rangle\left\langle\phi_{\ell}^{p} \mid \psi\right\rangle$. Here, $\ell$ and $p$ are the azimuthal and radial mode indices, respectively. Our goal is to predict the reflected state, from which we can derive the spatial mode scattering matrix $\hat{M}_{\lambda}$, which has as elements the spatial Fresnel coefficients (SFCs)

$$
c_{\ell, p, \ell^{\prime}, p^{\prime}}^{\lambda} \equiv\left\langle\phi_{\ell^{\prime}}^{p^{\prime}}\left|\hat{M}_{\lambda}\right| \phi_{\ell}^{p}\right\rangle
$$

To obtain these coefficients, we use two properties of LG modes: (i) $\phi_{\ell}^{p}(-x, y, z)=(-1)^{\ell} \phi_{-\ell}^{p}(x, y, z)$ and (ii) the known spatial derivatives of LG modes, $\frac{\partial \phi_{\ell}^{p}}{\partial x}$ and $\frac{\partial \phi_{\ell}^{p}}{\partial y}$, up to first order in $\theta_{0}\left(=\lambda / \pi \omega_{0}\right)$, the mode half-opening angle. We find the following first-order coefficients $c_{\ell, p, \ell^{\prime}, p^{\prime}}^{\lambda}$ for $\ell_{<}^{\gtrless}$ :

\begin{tabular}{|c|c|c|c|}
\hline$p^{\prime}$ & $-\ell \mp 1$ & $-\ell$ & $-\ell \pm 1$ \\
\hline$p-1$ & $\pm Z_{\lambda}^{ \pm} \sqrt{p}$ & 0 & 0 \\
\hline$p$ & $\pm Z_{\lambda}^{ \pm} \sqrt{|\ell|+p+1}$ & $(-1)^{\ell}$ & $\mp Z_{\lambda}^{\mp} \sqrt{|\ell|+p}$ \\
\hline$p+1$ & 0 & 0 & $\mp Z_{\lambda}^{\mp} \sqrt{p+1}$ \\
\hline
\end{tabular}

Here, we have combined all occurring shifts, i.e., the longitudinal and transverse, in each case the spatial and angular variants, in a single complex number $Z_{\lambda}^{ \pm}=$ $\frac{\theta_{0}}{2^{3 / 2}}(-1)^{\ell}\left(X_{\lambda} \pm i Y_{\lambda}\right)$. This is specific to Laguerre-Gauss modes. Figure 2 shows exemplarily the SFC intensities $C_{\ell, \ell^{\prime}}^{p}=\left|c_{\ell, \ell^{\prime}}^{p}\right|^{2}$ for air-glass reflection $\left(\theta=70^{\circ}\right)$ of a $p$-polarized LG mode with $\theta_{0}=0.05$, assuming for now a purely azimuthal LG mode $(p=0)$. We find that reflection described by the SFCs induces a transformation of a pure $\{\ell\}$ mode into a superposition of $\{-\ell-1,-\ell$, $-\ell+1\}$ modes, where the minus sign stems simply from OAM reversal upon reflection. The coupling strength to the OAM sideband modes $\{-\ell-1,-\ell+1\}$ is proportional to $\theta_{0}^{2}$ and depends linearly on $\ell$. In our case of pure azimuthal modes, the coupling strength $C_{\ell, \ell^{\prime}}^{\lambda}$ is simply proportional to $\left(\theta_{0} \sqrt{|\ell|}\right)^{2}$; here, we recognize the effective mode opening angle which is proportional to $\theta_{0} \sqrt{|\ell|}$. We also see that the mode coupling is governed by intrinsic displacement induced by beam shifts $X_{\lambda}$ and $Y_{\lambda}$. In the particular case of external reflection at a dielectric, only angular beam shifts occur $[9,11]$; this can be seen by analyzing $X_{\lambda}$ and $Y_{\lambda}$ : for linear $s$ or $p$ polarization, $Y_{\lambda}$ vanishes, and $X_{\lambda}$ is purely imaginary-this corresponds to an angular shift within the plane of incidence. 
The discussion above was for the case of a single OAM input state; however, it is straightforward to extend this to an OAM superposition input state. The mode coupling by reflection is a coherent scattering process so that a superposition can be handled by decomposition into its constituent OAM modes.

As a demonstration experiment, we investigate the case displayed in Fig. 2, i.e., external reflection at an air-glass interface. As shown in Fig. 1, light from a single mode fiber pigtailed $635 \mathrm{~nm}$ laser is collimated using a $20 \times$ microscopy objective $M 1$ (beam waist $\omega_{0}=900 \mu \mathrm{m}$; this corresponds to $\left.\theta_{0}=0.0002\right)$. The light is sent to a spatial light modulator SLM1 (10 $\mu \mathrm{m}$ pixel size, $3 \mathrm{mrad}$ blaze angle), which imprints the desired azimuthal phase to produce the input OAM spectrum $\{\ell\}$. This beam is then focused, externally reflected at the hypotenuse plane of a standard BK7 $(n=1.52)$ glass prism, and then recollimated to a beam waist of $\omega_{0}=900 \mu \mathrm{m}$. For this telescope configuration, we use underfilled $10 \times$ microscopy objectives (to minimize aberrations) $M 3$ and $M 4$ (numerical aperture 0.25 ) to obtain $\theta_{0}=0.05$ inside the telescope, which is well within the paraxial approximation. The reflected beam $\mid$ out $\rangle$ is sent to SLM2 set to detect OAM index $\ell^{\prime}$ in combination with microscopy objective $M 4$ and a single mode fiber. The reflected-beam axis is adjusted to be the axis of the reflected s-polarized fundamental Gaussian beam. The output fiber is connected to a photodiode (PD); its photocurrent is proportional to $C_{\ell, \ell^{\prime}}^{\lambda}$. By scanning the OAM of the input $(\ell)$ and output $\left(\ell^{\prime}\right)$ modes, we map the matrix containing the spatial Fresnel coefficients.

As a side step, we note that we use standard phase-only fork holograms on the SLMs to imprint the desired azimuthal OAM phase $e^{i \ell \phi}$ onto the field (blaze angle $3 \mathrm{mrad}$ ).

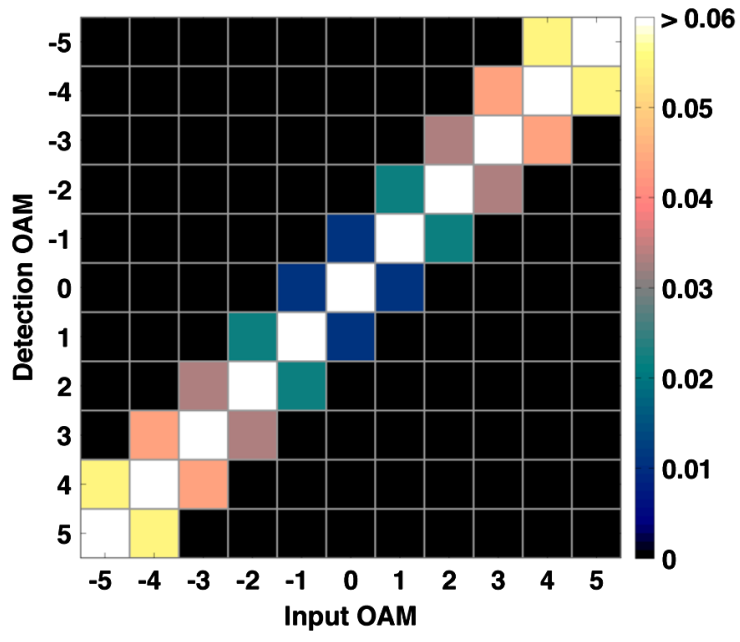

FIG. 2 (color online). Calculated SFC intensity $C_{\ell, \ell^{\prime}}^{p}=\left|c_{\ell, \ell^{\prime}}^{p}\right|^{2}$ for the case of $p$-polarized Laguerre-Gauss modes externally reflected at a dielectric interface $\left(\left(n=1.52, \theta=70^{\circ}, \theta_{0}=\right.\right.$ 0.05). Input $(\ell)$ and output $\left(\ell^{\prime}\right)$ OAM is shown on the horizontal and vertical axes, respectively (radial mode index $p=0$ ).
This transforms (for instance) a fundamental Gaussian mode not into a pure LG mode but into a superposition of LG modes, which have the same azimuthal index $\ell$ but different radial index $p$ [24]. In principle, this presents a problem since the radial modes suffer different transmission losses through the optical train. Therefore, we verify our experimental results by comparing with a fit-parameter-free numerical simulation [25], which not only accounts for spatial filtering but also for diffractive effects. We find that mode-dependent losses in the setup effectively lessen the influence of higher-order radial modes. As a bonus, this numerical method also enables validation of our analytical theory, as shown for the case $p=0$ [26].

Continuing now on the main line, we deduce from the measured $C_{\ell, \ell^{\prime}}^{\lambda}$ the relative intensity in the OAM sidebands compared to the total reflected intensity $I_{\text {rel }}^{\lambda}(\ell)=$ $\left(C_{\ell,-\ell-1}^{\lambda}+C_{\ell,-\ell+1}^{\lambda}\right) / \sum_{\ell^{\prime}} C_{\ell, \ell^{\prime}}^{\lambda}$. In order to improve the signal-to-noise ratio, we use polarization modulation $(s$ versus $p$ ) by rotating half-wave plates (WP1 and WP2) before and after reflection from the interface; this enables polarization-differential measurement. The experiment thus yields the polarization differential $I_{\mathrm{pd}}(\ell)=I_{\mathrm{rel}}^{p}(\ell)-$ $I_{\text {rel }}^{s}(\ell)$, which is plotted in Fig. 3 versus the input $\ell$, at a fixed angle of incidence $\left(\theta_{0}=70^{\circ}\right)$. The data for $\theta_{0}=0.0002$ have been obtained without the telescope. In this case, our theory predicts polarization-differential sidebands $<10^{-5}$ in the experimentally addressed $\ell$ range, which is much smaller than (but consistent with) the experimental accuracy. For $\theta_{0}=0.05$, the mode coupling is increased, in good agreement with theory. From experiments, as well as from numerical simulation, we

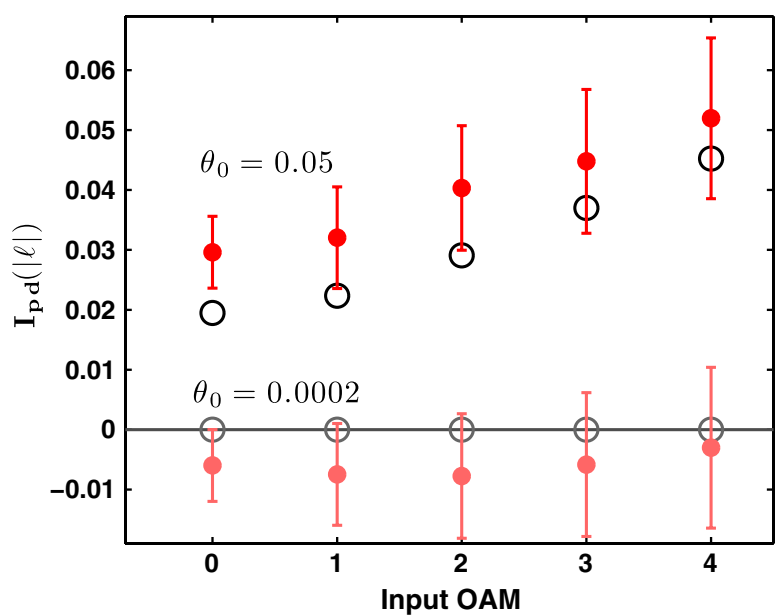

FIG. 3 (color online). Experimental (solid circles) and numerical (open circles) polarization-differential SFC sideband intensity $I_{\mathrm{pd}}(\ell)$. For an input beam with $\ell$ (horizontal axis), it shows the sum of the two OAM sidebands (with $-\ell-1$ and $-\ell+1$ ) appearing after external reflection by an air-glass interface, at an angle of incidence $\theta=70^{\circ}$, for two values of the beam spread $\theta_{0}$. Error bars are estimated from multiple measurement runs to take mechanical drifts and misalignment into account. 


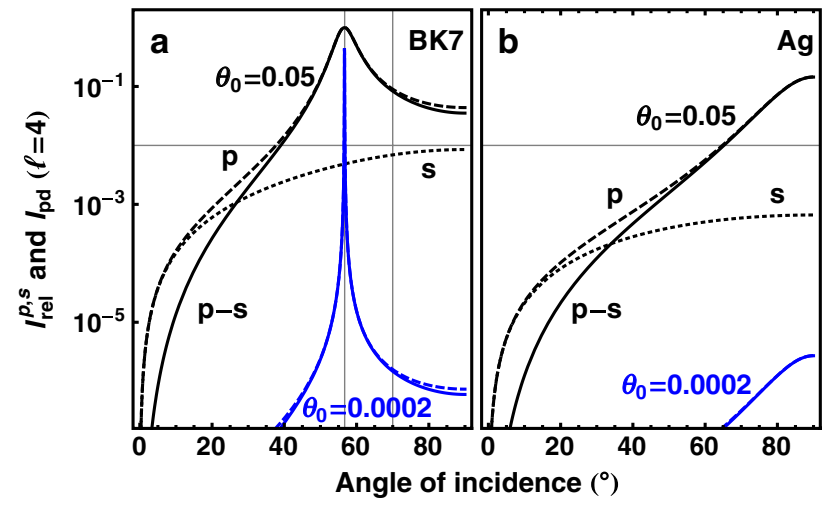

FIG. 4 (color online). The relative $I_{\text {rel }}^{p, s}$ and polarizationdifferential $I_{\mathrm{pd}}$ intensity for $(\ell=4, p=0)$ of the OAM sidebands (theory, logarithmic plots) for external reflection. The left panel (a) shows the case for an air-BK7 interface $(n=1.52)$, and (b) that for a silver mirror; the lower blue curves represent $\theta_{0}=0.0002$ ("collimated"), and the upper black curves represent $\theta_{0}=0.05$. We see that in the case of the glass interface, the polarization-differential sideband intensity (solid curve) is dominated by $p$ polarization (dashed curve), since $s$ polarization (dotted line) does not experience the Brewster resonance. The vertical lines indicate the Brewster angle and the angle for which we show measurements in Fig. $3\left(70^{\circ}\right)$.

observe that mode coupling to $-\ell \pm 2$ modes is at least an order of magnitude smaller than the coupling to $-\ell \pm 1$.

In Fig. 4(a), we present calculations of the SFC sidebands for the full range of angles of incidence. We use a pure LG mode with $\ell=4$ and $p=0$ as input beam and display the data for $s$ and $p$ polarization separately, as well as for the polarization-differential case. For $p$ polarization, a Brewster resonance occurs due to the vanishing of the reflection, making its contribution to the sidebands in most cases much larger than that of $s$ polarization; i.e., the polarization differential $I_{\mathrm{pd}}(\ell)$ is a sensible measure. For $\theta_{0}=0.0002$, the "neighboring" $\ell=3$ and $\ell=5$ sidebands are (much) weaker than 0.01 , except in a very narrow $\left(0.5^{\circ}\right)$ angular window centered at the Brewster angle.

It is interesting to compare our results for an air-glass interface with other cases, such as an air-metal interface. Our theory is fully adequate for this since the material properties enter only via the refractive index (or dielectric constant) that is complex-valued for a metal. Figure 4(b) gives the SFC sidebands $I_{\mathrm{pd}}(\ell=4)$ for a silver interface $\left(n_{\mathrm{Ag}}=0.14+4 i\right.$ at $\left.\lambda=635 \mathrm{~nm}\right)$. In the collimated case, the sideband intensity is now much smaller $\left(<10^{-5}\right.$ at all angles of incidence) than for an air-glass interface, basically since a metal is a much better reflector. Also, the Brewster resonance is absent in this case. We expect similar results for a dielectric Bragg mirror. It will be interesting to check these predictions experimentally.

In conclusion, we have introduced the concept of spatial Fresnel coefficients (SFCs) to describe transverse-modedependent reflection of a light beam. In the OAM basis, we find that an OAM mode $\{\ell\}$ acquires sidebands. The sidebands are due to first-order diffractive corrections to geometric optics (GH and IF effects); these lead to modedependent displacement and thus to coupling to "neighboring" OAM modes. We find that these effects scale with the angular spread of the beam. The sidebands disappear only in the limit of a completely reflecting mirror with an infinite refractive index step. Such mirrors do not exist, so in practice sidebands do occur. Nevertheless, for a wellcollimated beam, the sidebands are small; this bodes well for the use of folding mirrors or beam splitters in optical setups in the laboratory and for mirror-assisted free-space OAM communication. However, already for a moderately focused beam $\left(\theta_{0}=0.05\right)$ the sidebands become measurable (relative intensity of a few \%) and have to be accounted for in any experiment involving partial reflection of OAM beams.

An intriguing question is, can we undo the SFC-induced mode coupling? The reflective scattering process described by the matrix $c_{\ell, \ell^{\prime}}^{\lambda}$ is reversible, so that in principle superposition input states can be recovered with unity fidelity. But how can this be achieved experimentally? An ordinary mirror cannot do this since it simply adds to the diffractive beam shifts (GH and IF). We thus need an optical device that cancels intrinsic beam shifts; possibly, a negativeindex metamaterial [27] or photonic crystal [28] could achieve this task.

We acknowledge fruitful discussions with M.J.A. de Dood, J. B. Götte, and G. Nienhuis, and financial support by NWO and the EU STREP program 255914 (PHORBITECH).

*loeffler@physics.leidenuniv.nl

[1] L. Allen, M. W. Beijersbergen, R. J. C. Spreeuw, and J. P. Woerdman, Phys. Rev. A 45, 8185 (1992).

[2] A. Mair, A. Vaziri, G. Weihs, and A. Zeilinger, Nature (London) 412, 313 (2001).

[3] H. Bechmann-Pasquinucci and W. Tittel, Phys. Rev. A 61, 062308 (2000).

[4] G. Gibson, J. Courtial, M. Padgett, M. Vasnetsov, V. Pas'ko, S. Barnett, and S. Franke-Arnold, Opt. Express 12, 5448 (2004).

[5] Only the eigenstates, $s$ or $p$ polarization, remain pure upon reflection, albeit attenuated.

[6] F. Goos and H. Hänchen, Ann. Phys. (Leipzig) 436, 333 (1947).

[7] F. I. Fedorov, Dokl. Akad. Nauk SSSR 105, 465 (1955).

[8] C. Imbert, Phys. Rev. D 5, 787 (1972).

[9] K. Y. Bliokh and Y. P. Bliokh, Phys. Rev. Lett. 96, 073903 (2006).

[10] O. Hosten and P. Kwiat, Science 319, 787 (2008).

[11] A. Aiello and J. P. Woerdman, Opt. Lett. 33, 1437 (2008).

[12] K. Y. Bliokh and Y. P. Bliokh, Phys. Rev. E 75, 066609 (2007).

[13] M. Merano, A. Aiello, M.P. van Exter, and J.P. Woerdman, Nature Photon. 3, 337 (2009). 
[14] M. V. Vasnetsov, V. A. Pas'ko, and M. S. Soskin, New J. Phys. 7, 46 (2005).

[15] R. Zambrini and S. M. Barnett, Phys. Rev. Lett. 96, 113901 (2006).

[16] H. Okuda and H. Sasada, Opt. Express 14, 8393 (2006).

[17] H. Okuda and H. Sasada, J. Opt. Soc. Am. A 25, 881 (2008).

[18] M. Dennis and J. Götte, arXiv:1205.6457 [Phys. Rev. Lett. (to be published)].

[19] M. R. Dennis and J. B. Götte, New J. Phys. 14, 073013 (2012).

[20] A. Aiello and J. P. Woerdman, Phys. Rev. A 70, 023808 (2004).

[21] K. Y. Bliokh, I. V. Shadrivov, and Y. S. Kivshar, Opt. Lett. 34, 389 (2009).
[22] M. Merano, N. Hermosa, J. P. Woerdman, and A. Aiello, Phys. Rev. A 82, 023817 (2010).

[23] A. Aiello, New J. Phys. 14, 013058 (2012).

[24] N. R. Heckenberg, R. McDuff, C.P. Smith, H. Rubinsztein-Dunlop, and M.J. Wegener, Opt. Quantum Electron. 24, S951 (1992).

[25] The field is propagated by the Kirchhoff integral and reflected at the interface using the Debye approximation; exact dimensions are given in Fig. 3.

[26] See Supplemental Material at http://link.aps.org/ supplemental/10.1103/PhysRevLett.109.113602 for numerical simulation of the experiment, as well as for validation of our theory of spatial Fresnel coefficients.

[27] P. R. Berman, Phys. Rev. E 66, 067603 (2002).

[28] J. He, J. Yi, and S. He, Opt. Express 14, 3024 (2006). 